Pacific Journal of Mathematics

NRC PUTTING ALGEBRAS FOR 


\title{
GENERIC SPLITTING ALGEBRAS FOR PIC
}

\author{
GERALD GARFINKEL
}

Our purpose is to develop a splitting theory for the functor Pic. For each rank one projective module $P$, we exhibit a generic splitting algebra $T_{\infty}(P / R)$. Whenever $n$ is a multiple of the order of $P$ in $\operatorname{Pic}(R)$, it has a splitting algebra which is a rank $n$ projective module. We then show the relationship of the latter algebra to the usual splitting ring of an ideal in a Dedekind domain.

Throughout this paper all rings and algebras are commutative and associative and have identities. The symbol " $R$ " will always denote a fixed but arbitrary ring and all modules and algebras are over $R$. An unadorned tensor product sign " $\otimes$ " is to be interpreted as " $\otimes_{R}$ ". If $M$ is an $R$-module we let $M^{n}$ be $M \otimes \cdots \otimes M(n$ times), $M^{*}$ be $\operatorname{Hom}(M, R)$ and $e: M \otimes M^{*} \longrightarrow R$ be the evaluation map (i.e., $\left.e^{\prime} x \otimes f\right)=f(x)$ for all $x \in M$ and $f \in M^{*}$ ).

An $\mathrm{R}$-module $P$ is a rank $n$ projective if it is finitely generated and if for each prime ideal $\mathfrak{m}$ of $R, P_{\mathfrak{m}}$ is a free $R_{\mathfrak{m}}$ module of rank $n$. If $P$ is a rank one projective, so is $P^{*}$ and $e: P \otimes P^{*} \longrightarrow R$ is an isomorphism. Thus Pic $(R)$, the set of isomorphism classes $(P)$ of rank one projectives $P$, is a group under the multiplication rule: $(P)(Q)=(P \otimes Q)$. If $P$ is a rank one projective we write $P^{-n}$ for $P^{* n}\left(\cong P^{n *}\right)$. An $R$-algebra $S$ splits $(P)$ in Pic $(R)$ if $P \otimes S \cong S$ as $S$-modules. See [3] for more details.

A final note on general notation: If $\left\{A_{i}\right\}$ is a collection of $R$ modules, by $\sum \oplus A_{i} U^{i}$ is meant the $R$-module whose elements are all finite sums $\sum a_{i} U^{i}$ where $a_{i} \in A_{i}$. Addition and scalar multiplication are defined as for polynomials in the indeterminant $U$. Sometimes the $U$ may be in lower case and it may or may not have a subscript. Clearly the notation is such that $\sum \oplus R U^{i}$ is the module of ordinary polynomials over $R$ in the indeterminant $U$.

In $\S 2$ we define for each rank one projective $P$ an algebra $T_{\infty}(P / R)$ which by Theorem 1 is a generic splitting algebra for the module $Q$ in the same sense that $F_{m}(A)$ is a generic splitting algebra for the central simple algebra $A$ in the works of Amitsur [1] and Roquette [8]. If $T^{\prime}(P / R)$ is the tensor algebra of $P$, Theorem 2 characterizes the splitting algebras of $P$ as those commutative algebras $S$ such that $S \otimes T^{\prime}(P / R)$ is isomorphic to $S[X]$.

In $\S 3$ it is shown that for every isomorphism $f: P^{n} \longrightarrow R, P$ has a splitting algebra $T_{n}(P / R, f)$ which is a rank $n$ projective module. This algebra is separable if and only if $n$ is a unit of $R$. We close 
the section with some information about when two of the generic or finite splitting algebras are isomorphic (as graded algebras).

We define Picard ideals as those which are rank one projective modules. Our last section is concerned with the case in which $I$ is a Picard ideal with $I^{n}=R w$ and $T=T_{n}\left(I / R, w^{-1}\right)$. We give some information about when $T$ is a domain and in Theorem 4 show that if $R$ is Dedekind and $n$ is both the order of $(I)$ in Pic $(R)$ and is a unit of $R$, then $T$ is the usual splitting algebra for $I$. We give two examples illustrating what may happen to $T$ when $R$ is Dedekind but $n$ fails to satisfy the hypotheses of Theorem 4 .

2. Generic splitting algebras. Throughout this section $P$ is a rank one projective. Before we can define the generic splitting algebra we need the following rather technical lemma.

Lemma 1. (i) Suppose $\alpha$ is any permutation of the first $n$ integers and let $\theta: P^{n} \longrightarrow P^{n}$ be the unique homomorphism with $\theta\left(x_{1} \otimes \cdots \otimes x_{n}\right)=x_{\alpha_{1}} \otimes \cdots \otimes x_{\alpha n}$. Then $\theta$ is the identity map.

(ii) If $m$ and $n$ are any two integers, the various maps from $P^{n} \otimes P^{m}$ to $P^{n+m}$ induced by interchanging factors and applying the evaluation map $e$ and the identity map are all the same. We denote this map by $t_{n, m}: P^{n} \otimes P^{m} \longrightarrow P^{n+m}$.

(iii) If $\sigma: P^{n} \otimes P^{m} \longrightarrow P^{m} \otimes P^{n}$ is the "switch" map, then $t_{m, n} \cdot \sigma=t_{n, m}$.

(iv) If $q$ is also an integer, the following diagram commutes:

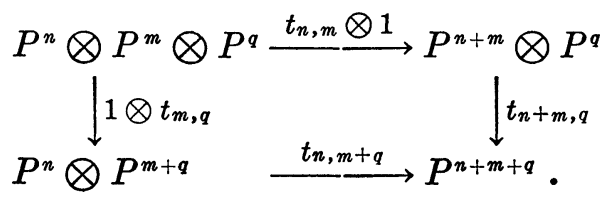

Proof. For (i) we need only show that Ifor each maximal ideal $\mathfrak{m}$, the map $\theta_{m}: P_{\mathfrak{m}}^{n} \longrightarrow P_{\mathfrak{m}}^{n}$ is the identity. Since $R_{\mathfrak{m}}$ is local, $P_{\mathfrak{m}}=$ $R_{\mathfrak{m}} x$ for some $x$ in $P_{\mathfrak{m}}$. Thus $P_{\mathfrak{m}}^{n}=R_{\mathfrak{m}} y$ where $y=x \otimes \cdots \otimes x$ and clearly $\theta_{\mathfrak{m}}(y)=y$. The proofs of the other parts of the lemma are clear from the statement and proof of (i).

We can now make the following definition.

Definition. Suppose $(P)$ is in Pic $(R)$, let $T_{\infty}(P / R)=\sum_{i=-\infty}^{+\infty} \oplus P^{i} U^{i}$ as an $R$-module, and define a multiplication by:

$$
\left(\sum a_{i} U^{i}\right) \cdot\left(\sum b_{j} U^{j}\right)=\sum_{i, j} t_{i, j}\left(a_{i} \otimes b_{j}\right) U^{i+j} .
$$


Lemma 1 shows that $T_{\infty}(P / R)$ is a commutative, associative $R$ algebra. Let $T^{\prime}(P / R)=\sum_{i=0}^{\infty} P^{i} U^{i}$. Clearly $T^{\prime}(P / R)$ is an $R$-subalgebra of $T_{\infty}(P / R)$ which is isomorphic to the tensor algebra of the $R$-module $P$.

REMARK. It is clear that up to isomorphism $T_{\infty}(P / R)$ depends only on the isomorphism class of the $R$-module $P$ and if $S$ is any commutative $R$-algebra, then $S \otimes T_{\infty}(P / R) \cong T_{\infty}(S \otimes P / S)$. Similar facts hold for $T^{\prime}$. In fact $T_{\infty}(P / R)$ and $T^{\prime}(P / R)$ are both natural in the following sense. For any commutative ring $R$, let $P C A(R)$ be the set of isomorphism classes of commutative $R$-algebras which are projective and countably generated as $R$-modules. If $\mathscr{K}$ is the category of commutative rings with identity and $\mathscr{S}$ the category of Sets, then Pic and $P C A$ can be considered functors from $\mathscr{K}$ to $\mathscr{S}$ and $T$ and $T^{\prime}$ then induce natural transformations from Pic to $P C A$.

Proposition 1. If $S$ is any commutative R-algebra splitting $P$, then $S \otimes T_{\infty}(P / R) \cong S[Z]$ (the group ring over $S$ of the additive group of integers $Z) \cong S\left[X, X^{-1}\right]$ the polynomial ring over $S$ in the indeterminants $X$ and $\left.X^{-1}\right)$. Also $S \otimes T^{\prime}(P / R) \cong S[X]$.

Proof. By the above remark,

$$
S \otimes T_{\infty}(P / R) \cong T_{\infty}(S / S)=\sum_{i=-\infty}^{\infty} S U^{i}
$$

Multiplication in the latter is defined by

$$
\left(\sum a_{i} U^{i}\right)\left(\sum b_{j} U^{j}\right)=\sum a_{i} b_{j} U^{i+j},
$$

and thus it is clearly isomorphic to $S[Z]$ and to $S\left[X, X^{-1}\right]$. A similar calculation shows $S \otimes T^{\prime}(P / R) \cong S[X]$.

Proposition 2. $T=T_{\infty}(P / R)$ splits $P$; in fact if we let $\theta: T \otimes P \rightarrow T$ be the map induced by the maps

$$
t_{n, 1}: P^{n} U^{n} \otimes P \longrightarrow P^{n+1} U^{n+1},
$$

then $\theta$ is a T-isomorphism.

Proof. Clear.

In fact not only does $T$ split $P$, but $T$ is a "generic" splitting algebra for $P$ in the sense of the following theorem.

Theorem 1. Let $(P)$ be in Pic $(R)$ and $T=T_{\infty}(P / R)$. Then for 
any commutative $R$-algebra $S$, the following are equivalent:

(i) $S$ splits $P$,

(ii) $S \otimes T \cong S[Z] \cong S\left[X, X^{-1}\right]$ as S-algebras,

(iii) there is an $R$-algebra homomorphism from $T$ to $S$.

Proof. (i) $\Rightarrow$ (ii) by Proposition 1. If (ii) holds, then the composition $T \longrightarrow S \otimes T \cong S[Z] \longrightarrow S$ is an $R$-algebra map where the last factor in the composition is induced by the group homomorphism from $\boldsymbol{Z}$ to the one element group. Thus (ii) $\Rightarrow$ (iii). If (iii) holds we can view $S$ as a $T$-algebra. Then since by Proposition 2, $T$ splits $P$, we have

$$
S \otimes P \cong S \otimes_{T} T \otimes P \cong S \otimes_{T} T \cong S
$$

as $S$-modules. Hence (iii) $\Longrightarrow$ (i).

Using the map $\theta$ of Proposition 2 it is easy to see that $P^{\prime}=$ $\sum_{i=1}^{\infty} P^{i} U^{i}$ is a $T^{\prime}$ ideal isomornhic to $T^{\prime \prime} \otimes P$. Thus $T^{\prime}$ splits $P$ if and only if $P^{\prime}$ is $T^{\prime}$-free. The following theorem shows that $T^{\prime}$ does not split $P$ (except when $P$ is itself trivial) and that moreover the "splitting" algebras for $P, T^{\prime}$ and $P^{\prime}$ are the same.

THEOREM 2. For each commutative algebra $S$ the fnllowing are equivalent:

(i) $S$ splits $P$,

(ii) $S \otimes T^{\prime}(P / R) \cong S\lceil X\rceil$ as S-algebras,

(iii) $S \otimes P^{\prime}$ is a freo, $S \otimes T^{\prime}(P / R)$ module.

Prnof. By the naturality of $T^{\prime}=T^{\prime}(P / R)$ we can assume $S=$ $R$. (i) $\Rightarrow$ (ii): By Proposition 1. (ii) $\Rightarrow$ (iii): Let $\varphi: T^{\prime} \rightarrow R$ be defined by $\varphi\left(\sum a_{i} U^{i}\right)=a_{0}$. Clearly $\varphi$ is an $R$-algebra map with kernel $P^{\prime}$. Suppose $\alpha: R\lceil X\rceil \longrightarrow T^{\prime}$ is any $R$-isomorphism. Then since the kernel of $\varphi \alpha$ is generated by $y=X-\varphi \alpha X$, it is easy to see that $P^{\prime}$ is generated by $\alpha y$ and thus is a free $T^{\prime}$ module. (iii) $\Rightarrow$ (i): If inc: $R \longrightarrow T^{\prime}$ is the inclusion map, then since $\varphi$ inc is the identity map of $R$, Pic (inc) is a split monomorphism. Since $\left(P^{\prime}\right)$ is Pic (inc) of $(P)$, we see that $P^{\prime}$ is $T^{\prime}$-free if and only if $P$ is $P$-free.

3. Finite splitting algebras. Throughout this section, $P$ is a rank one projective module.

Definition. If $f: P^{n} \longrightarrow R$ is an isomorphism, we let $T_{n}(P / R, f)$ be the $R$-module $\sum_{i=0}^{n-1} \oplus P^{i} u_{f}^{i}$. We define a multiplication on $T_{n}(P / R, f)$ by the formula: 


$$
\left(a u_{f}^{i}\right)\left(b u_{f}^{j}\right)=\left\{\begin{array}{cc}
a b u_{f}^{i+j} & \text { when } i+j<n \\
f a b u_{f}^{i+j-n} & \text { when } i+j \geqq n
\end{array}\right.
$$

and the distributive law: $\left(\sum_{i} a_{i} u_{f}^{i}\right)\left(\sum_{j} b^{j} u_{f}^{j}\right)=\sum_{i, j}\left(a_{i} u_{f}^{i}\right)\left(b_{j} u_{f}^{j}\right)$. We will often write " $T_{n}$ " for " $T_{n}(P / R, f)$ " and " $u^{i}$ " for " $u_{f}^{i}$ ".

Note that an isomorphism $f: P^{n} \longrightarrow R$ is an element of $P^{n *}=$ $P^{-n}$. Suppose $f(w)=1$. If for each $k \geqq 0$, we let $w^{-k}$ be the element $f^{k}$ in $P^{-n k}$, then for each integer $k, P^{n k}$ is the free $R$-module with generator $w^{k}$. Thus each element $Y$ of $T=T_{\infty}(P / R)$ is uniquely expressible as

$$
Y=\sum_{j=0}^{n-1} \sum_{k=-\infty}^{+\infty} a_{k, j} w^{k} U^{k n+j} \text { with } a_{k, j} \in P^{j} .
$$

Proposition 3. Suppose $f, w, T$ and $T_{n}$ are as above. Then there is an $R$-module map $\pi: T \longrightarrow T_{n}$ such that

(i) $\pi$ is an $R$-module epimorphism

(ii) $\pi$ is multiplicative

(iii) kernel $\pi=T\left(w U^{n}-1\right)=T\left(f U^{-n}-1\right)$

(iv) if $\pi^{\prime}$ is $\pi$ restricted to $T^{\prime}=T^{\prime}(P / R)$ then $\pi^{\prime}$ is an $R$-module epimorphism with kernel $T^{\prime}\left(w U^{n}-1\right)$.

In particular if $I=T\left(w U^{n}-1\right)$ and $I^{\prime}=T^{\prime}\left(w U^{n}-1\right)$, then $T_{n}$ is an R-algebra which is isomorphic to $T / I$ and to $T^{\prime} / I^{\prime}$.

Proof. If $Y$ is a nonzero element of $T$ of the form $(*)$, we define $\pi(Y)=\sum_{j, k} a_{k, j} u_{f}^{j}$. From this definition it is clear that $\pi$ is a well defined $R$-module epimorphism. The fact that when $j+j^{\prime} \geqq$ $n$ then $a_{k, j} a_{k^{\prime}, j^{\prime}}=f\left(a_{k, j} a_{k^{\prime}, j^{\prime}}\right) w$, shows that $\pi$ is multiplicative. Thus conditions (i) and (ii) hold.

Now let $I=T\left(w U^{n}-1\right)$. Since $-f U^{-n}\left(w U^{n}-1\right)=f U^{-n}-1$ and $\left(-w U^{n}\right)\left(f U^{-n}-1\right)=w U^{n}-1$, we see that $I=T\left(f U^{-n}-1\right)$ also. Since $\pi\left(w U^{n}-1\right)=0$, it is clear that $I$ is contained in kernel $\pi$. Now suppose $Y$ is an element of form $(*)$. Then it is easy to see that $\pi(Y)=0$ if and only if for each $0 \leqq j<n$, we have $\sum_{k} a_{k, j}=0$. Thus if $\pi(Y)=0$, then $Y=\sum_{j, k} a_{k, j} U^{j}\left(w^{k} U^{n k}-1\right)$. However, if $k>0$, then $\left(w U^{n}\right)^{k}-1=\left(w U^{n}-1\right)\left(1+w U^{n}+\cdots+\left(w U^{n}\right)^{k-1}\right)$ and hence is in $I$. A similar equation shows that if $k<0$, then

$$
\left(w U^{n}\right)^{k}-1=\left(f U^{-n}\right)^{-k}-1
$$

is also in $I$. Hence condition (iii) has been verified.

Clearly $\pi^{\prime}$ is an epimorphism with kernel $I \cap T^{\prime}$. To prove (iv) we must show that $I \cap T^{\prime}$ is contained in $T^{\prime}\left(w U^{n}-1\right)$. An element 
$Y$ of $I \cap T^{\prime}$ is of the form $Y=\sum_{M}^{N} a_{i} U^{i}\left(U^{0}-w U^{n}\right)$ where $M \leqq N$ and each $a_{i} \in P^{i}$. However, since $a_{M} U^{M}$ is the homogeneous component of $Y$ of lowest degree and $Y$ is in $T^{\prime}$, we see that $M \geqq 0$ and hence $Y$ is in $T^{\prime}\left(w U^{n}-1\right)$.

The last statement in the proposition is clear.

Notation. If $M$ is any $R$-module, let $E^{0}(M / R)=R$, and if $n>0$ let $E^{n}(M / R)=M \wedge \cdots \wedge M$ (the $n$-th exterior product of $M$ with itself).

Lemma 2. Suppose $M$ is any $R$-module, $(P)$ is in Pic $(R)$ and $n>0$ is an integer. Then there is an $R$-module isomorphism

$$
\theta: P^{n} \otimes E^{n}(M / R) \longrightarrow E^{n}(P \otimes M / R)
$$

such that if $a_{i} \in P, x_{i} \in M$ for $i \leqq n$,

$$
\theta\left(\left(a_{1} \otimes \cdots \otimes a_{n}\right) \otimes\left(x_{1} \wedge \cdots \wedge x_{n}\right)\right)=\left(a_{1} \otimes x_{1}\right) \wedge \cdots \wedge\left(a_{n} \otimes x_{n}\right) .
$$

Proof. Let $M^{\prime}=M \times \cdots \times M$ (cartesian product of $M$ with itself $n$ times) and $P^{\prime}=P \times \cdots \times P$ and define

$$
\theta: P^{\prime} \times M^{\prime} \longrightarrow E^{n}(P \otimes M / R)
$$

by $\theta^{\prime}(a, x)=\left(a_{1} \otimes x_{1}\right) \wedge \cdots \wedge\left(a_{n} \otimes x_{n}\right)$ when $a=\left(a_{1}, \cdots, a_{n}\right) \in P^{\prime}$ and $x=\left(x_{1}, \cdots, x_{n}\right) \in M^{\prime}$. Clearly $\theta^{\prime}$ is multilinear in the variables $a_{1}, \cdots, x_{n}$. For each maximal ideal $\mathfrak{m}$ of $R$, let $f_{\mathfrak{n}}$ be the map

$$
E^{n}(P \otimes M / R) \longrightarrow R_{\mathfrak{m}} \otimes E^{n}(P \otimes M / R) \longrightarrow E^{n}\left(P_{\mathfrak{m}} \otimes M_{\mathfrak{m}} / R_{\mathfrak{m}}\right)
$$

induced by localization. It is easy to check that each $f_{\mathfrak{m}} \theta^{\prime}$ is alternating in the $x_{i}$ 's and thus $\theta^{\prime}$ is alternating in those variables. Hence there is a well defined homomorphism $\theta$ satisfying the given property. Since each $\theta_{\mathfrak{m}}$ is an isomorphism, $\theta$ itself is an isomorphism.

Theorem 3. Suppose $(P)$ is in Pic $(R)$ and $n>0$. Then the following are equivalent:

(i) $(P)^{n}=1$,

(ii) there is a commutative $R$-algebra $T$ which is a rank $n$ projective $R$-module with $T \otimes P \cong T$ as $T$-modules,

(iii) there is a rank $n$ projective $R$-module $M$ with $M \otimes P \cong M$.

Proof. (A slightly weaker form of (i) $\Leftrightarrow$ (iii) is shown by Bass [2].)

(i) $\Rightarrow$ (ii): By Propositions 2 and 3 , we can take $T=T_{n}(P / R, f)$ where $f: P^{n} \longrightarrow R$ is any isomorphism.

(ii) $\Rightarrow$ (iii): Let $M=T$ and forget the $T$-structure. 
(iii) $\Rightarrow$ (i): $\quad$ By [3, p. 142], $E^{n}(M / R)=E^{n}(P \otimes M / R)=N$ is a rank one projective $R$-module. However, by Lemma $2, P^{n} \otimes N \cong N$. Thus $(P)^{n}(N)=(N)$ in Pic $(R)$ and since Pic $(R)$ is a group, $(P)^{n}=1$.

Proposition 4. Suppose $(P)$ is in Pic $(R)$. Then $T^{\prime}(P / R)$ and $T_{\infty}(P / R)$ are not separable $R$-algebras. If $f: p^{n} \longrightarrow R$ is an isomorphism, then $T_{n}(P / R, f)$ is a separable $R$-algebra if and only if $n$ is a unit in $R$.

Proof. The first statement follows from Villameyer's Theorem [7] which states that a separable, projective algebra is also finitely generated as an $R$-module and clearly $T^{\prime}$ and $T_{\infty}$ are not finitely generated. By Bass [2; pp. 94, 96] $T_{n}=T_{n}(P / R, f)$ is separable if and only if for all maximal ideals $\mathrm{m}$ of $R,(R / \mathfrak{m}) \otimes T_{n}$ is a separable $R / \mathrm{m}$-algebra. By the naturality of $T_{\infty}$ and Proposition 3 , it is clear that if $\bar{R}=R / \mathrm{m}$, then $\bar{R} \otimes T_{n}=\bar{R}[X] /\left(1-w X^{n}\right)$ for some element $w$ in $\bar{R}$. Thus $\bar{R} \otimes T_{n}$ is separable if and only if $X^{n}-w^{-1}$ is a separable polynomial in $\bar{R}[X]$; however this is true if and only if $n$ is a unit (i.e., nonzero) in $\bar{R}$. But clearly $n$ is nonzero in each $R / \mathrm{m}$ if and only if $n$ is not in any maximal ideal $\mathrm{m}$; which is to say that $n$ is a unit in $R$.

We consider an isomorphism between two graded algebras $A$ and $B$ to be graded (and write $A \cong_{g} B$ ) if it preserves homogeneous components. Noting that $T_{\infty}(P / R), T^{\prime}(P / R)$ and $T_{n}(P / R, f)$ are graded algebras with grading respectively $\boldsymbol{Z}, \boldsymbol{Z}^{+}$and $\boldsymbol{Z} / n \boldsymbol{Z}$, we can now state:

Proposition 5. Suppose $(P)$ and $(Q)$ are in Pic $(R)$.

(i) $T^{\prime}(Q / R) \cong_{g} T^{\prime}(P / R)$ if and only if $(Q)=(P)$.

(ii) The mapping $x U^{m} \longrightarrow x U^{-m}$ defines a graded isomorphism $h: T_{\infty}\left(P^{*} / R\right) \longrightarrow T_{\infty}(P / R)$. $\pm 1$

(iii) $T_{\infty}(Q / R) \cong{ }_{g} T_{\infty}(P / R)$ if and only if $(Q)=(P)^{e}$ where $e=$

(iv) There is a graded isomorphism $h: T_{m}(Q / R, g) \longrightarrow T_{n}(P / R, f)$ if and only if $m=n$ and there are an integer $k>0$ and an isomorphism $h^{\prime}: Q \longrightarrow P^{k}$ with $(k, m)=1$ and $g=f^{k} h^{\prime n}$.

Proof. (i), (ii) and (iii) are clear, so we only give the proof of (iv).

$(\Leftarrow)$ : The correspondence $x U^{r} \longrightarrow h^{\prime r}(x) U^{k r}$ induces an algebra $\operatorname{map} h^{\prime \prime}: T^{\prime}(Q / R) \longrightarrow T^{\prime}(P / R)$. Since $h^{\prime \prime}\left(1-g^{-1} U^{n}\right)=1-\left(f^{-1} U^{n}\right)^{k}$, an element of $T^{\prime}(P / R)\left(1-f^{-1} U^{n}\right)$, by Proposition 3 we see that $h^{\prime \prime}$ induces a graded algebra homomorphism $h: T_{n}(Q / R, g) \longrightarrow T_{n}(P / R, f)$. Since $(\mathrm{k}, n)=1, h$ is onto and thus is an isomorphism since its domain 
and range are rank $n$ projectives. Clearly $h$ is graded.

$(\Rightarrow)$ : Clearly $m=n$ and since $h$ is a graded isomorphism there is an integer $k$ and a map $h^{\prime}: Q \longrightarrow P^{k}$ with $h(x U)=h^{\prime}(x) U^{k}$. Thus $h\left((Q U)^{r}\right) \subseteq(P U)^{r^{\prime}}$ where $k r \equiv r^{\prime}(\bmod n)$. Since $h$ is onto, the equation $k r \equiv 1$ is solvable and thus $(k, n)=1$. Also $k r \equiv k$ if and only if $r \equiv 1$ and so $h(Q U)=P^{k} U^{k}$. Therefore $h^{\prime}$ is onto and hence is an isomorphism.

4. Picard ideals. In this section we are interested in the case where the rank one projective module is actually an ideal of $R$-which we call a Picard ideal. In many rings (e.g., domains and Noetherian rings) this is always the case-i.e., Pic $(R)=C(R)$-the isomorphism classes of Picard ideals. See [3] or [9]. However by an exercise in Bourbaki [3, p. 179] it can be seen that $C(R)$ is not functoral, is not always a group and may be a proper subset of Pic $(R)$. Since $C(R)$ is not well behaved, one often gets information about it by studying the functor Pic $(R)$.

We introduce the following special notation for this section: " $I$ " will always denote an ideal of $R$ and " $K$ " is always the total quotient ring of $R . I^{n}$ is the ideal product of $I$ with itself $n$ times and $I^{(n)}=I \otimes \cdots \otimes I$. It is easy to see that if $I$ is flat then $I^{n} \cong I^{(n)}$. Finally we let $I^{-1}=\{x \in K: x I \subseteq R\}$.

Lemma 3. I is a Picard ideal with finite order in Pic $(R)$ if and only if some power of $I$ is generated by a non zero divisor. If either condition holds then the map $\theta: I^{-1} \longrightarrow I^{*}$ defined by $\theta(x)(a)=$ $x a$ is an isomorphism.

Proof. The necessity condition is clear. Now suppose $I^{n}=R w$ where $w$ is a non zero divisor. First note that $\varphi: I^{*} \longrightarrow I^{-1}$, defined by $\varphi(g)=g(w) w^{-1}$, is an inverse to $\theta$; which is thus an isomorphism. If $w=\sum a_{i} b_{i}$ with $a_{i} \in I$ and $b_{i} \in I^{n-1}$, it is easy to see that $\left\{a_{i}, \theta\left(b_{i}\right)\right\}$ is a finite projective basis for $I$ in the sense of [4, p. 132] and thus $I$ is finitely generated and projective as a module. Hence $I^{*}$ is flat and so $I \otimes I^{*} \cong I \cdot I^{*} \cong I I^{-1}$. Since $I \cdot I^{n-1} w^{-1}=R$, we see that $I \cdot I^{-1}=$ $R$. Then we can conclude that $I$ is rank one from the fact that $I \otimes I^{*} \cong R$. Clearly $(I)^{n}=1$.

Proposition 6. Let $I$ be an ideal of $R$ with $I^{n}=R w$ for some non zero divisor $w$ and let $K$ be the total quotient ring of $R$.

(i) $I$ is a Picard ideal and if we identify $w$ and $w^{-1}$ with multiplication by that element in $K$, then $w^{-1}: I^{n} \longrightarrow R$ and $w: I^{-n}$ $\longrightarrow R$ are isomorphisms.

(ii) The inclusions $R \subseteq I^{-1} \subseteq K$ induce inclusion maps 


$$
R[U] /\left(U^{n}-w\right) \longrightarrow T_{n}\left(I^{-1} / R, w\right) \longrightarrow K[U] /\left(U^{n}-w\right) .
$$

(iii) If $R$ is an integral domain, then $T_{n}\left(I / R, w^{-1}\right)$ is an integral domain if and only if $U^{n}-w$ is an irreducible polynomial over $K$.

Proof. (i) Clear from Lemma 3.

(ii) Clearly the inclusions $R \cong I^{-1} \subseteq K$ induce inclusions

$$
T^{\prime}(R / R) \subseteq T^{\prime}\left(I^{-1} / R\right) \subseteq T^{\prime}(K / K) \text {. }
$$

Thus if we define

$$
\begin{aligned}
& J_{0}=T^{\prime}(K / K)\left(w^{-1} U^{n}-1\right) \\
& J_{1}=T^{\prime}\left(I^{-1} / R\right) \cap J_{0} \\
& J_{2}=T^{\prime}(R / R) \cap J_{0}
\end{aligned}
$$

then there is a sequence of inclusions:

$$
R[U] / J_{2} \longrightarrow T^{\prime}\left(I^{-1} / R\right) / J_{1} \longrightarrow K[U] /\left(U^{n}-w\right) .
$$

Thus to prove (ii) we need only show that

$$
J_{1}=J_{1}^{\prime}=T^{\prime}\left(I^{-1} / R\right)\left(w^{-1} U^{n}-1\right)
$$

and $J_{2}=J_{2}^{\prime}=R[U]\left(U^{n}-w\right)$. Also it is clear that $J_{1}^{\prime} \subseteq J_{1}$ and since $U^{n}-w=w\left(w^{-1} U^{n}-1\right) \in J_{2}, J_{2}^{\prime} \subseteq J_{2}$. Thus we need only show the opposite inclusions.

Suppose $y=\left(\sum y_{i} U^{i}\right)\left(w^{-1} U^{n}-1\right)=\sum a_{i} U^{i}$ is in $J_{0}$. Note that $y_{i}=w\left(a_{i+n}+y_{i+n}\right)$. Now assume $y$ is in $J_{1}$, i.e., each $a_{i} \in I^{-i}$. If $y$ is not in $J_{1}^{\prime}$, let $N$ be the largest integer with $y_{N}$ not in $I^{-N}$. Then $y_{N}=w\left(a_{N+n}+y_{N+n}\right) \in I^{n} \cdot I^{-N-n}=I^{-N}$, a contradiction. Thus $J_{1}=J_{1}^{\prime}$. Now assume $y$ is in $J_{2}$. We claim that for each $i, y_{i}$ is in $R w$. If not, letting $N$ be a maximal counter-example, the above expression for $y_{N}$ show it is in $w(R+R w)=R w$, a contradiction. Thus

$$
y=w\left(\sum w^{-1} y_{i} U^{i}\right) w^{-1}\left(U^{n}-w\right)
$$

is an element of $J_{2}^{\prime}$ and so $J_{2}^{\prime}=J_{2}$.

(iii) It is easy to see that $K^{\prime}=K[U] /\left(U^{n}-w\right)$ is the total quotient ring of $R[U] /\left(U^{n}-w\right)$ and thus $K^{\prime}$ is also the total quotient ring of $T_{n}^{\prime}=T_{n}\left(I^{-1} / R, w\right)$. By Proposition 5 we see that $T_{n}^{\prime}$ is isomorphic to $T_{n}=T_{n}\left(I / R, w^{-1}\right)$. Thus $T_{n}$ is a domain if and only if $K^{\prime}$ is a field and it is well known that $K^{\prime}$ is a field if and only if $U^{n}-w$ is irreducible.

LEMMA 4. Suppose $n_{1}$ and $n_{2}$ are relatively prime positive integers and $w$ is an element of the field $K$. Then $X^{n_{1} n_{2}}-w$ is 
irreducible if and only if both $x^{n_{1}}-w$ and $x^{n_{2}}-w$ are irreducible.

Proof. Let $n=n_{1} n_{2}$. Clearly if $x^{n}-w$ is irreducible so is each $x^{n_{i}}-w$. Now suppose each $x^{n_{i}}-w$ is irreducible. Thus for each $i$, there is a field $L_{i}=K\left[u_{i}\right]$ where $x^{n_{i}}-w$ is the minimal polynomial of $u_{i}$. Since $\left(n_{1}, n_{2}\right)=1$, it is easy to see that any field compositum $L=L_{1} L_{2}$ is unique up to isomorphism and is of dimension $n$ over $K$. Suppose $s_{1}$ and $s_{2}$ are integers with $n_{1} s_{2}+n_{2} s_{1}=1$. Let $v$ be the element $u_{1}^{s_{1}} \cdot u_{2}^{s_{2}}$ in $L$. A simple calculation then shows that $v^{n_{1}}=u_{2}$ and $v^{n_{2}}=u_{1}$. Thus $v$ generates the field $L$ of dimension $n$ and satisfies the polynomial $v^{n}-w$. Hence $x^{n}-w$ is the minimal polynomial of $v$ and so is irreducible.

Theorem 4. Suppose $R$ is a Dedekind domain with quotient field $K$ and $I$ is an ideal of $R$ with $I^{n}=R w$, and $n$ is the order of $(I)$ in Pic $(R)$. Then $T=T_{n}\left(I / R, w^{-1}\right)$ is a domain. If $n$ is also a unit of $R$, then $T$ is a Dedekind domain and is isomorphic to the integral closure of $R$ in $K[X] /\left(X^{n}-w\right)$.

Proof. We first prove the theorem in the case where $n$ is nonzero in $R$. By Proposition 6, we need to show $f(X)=X^{n}-w$ is an irreducible $K$ polynomial. Let $g(X)$ be any irreducible factor of $f(X)$ of degree say $d$. We must show $d=n$.

Let $S$ be the integral closure of $R$ in $L=K[X] /(g(X))$. Since $n$ is a unit of $K, f(X)$ is a separable polynomial and hence so is $g(X)$. Since $L$ is a separable extension of $K, S$ is finitely generated as an $R$-module [6, p. 70]. Since $S$ is torsion free, it is also a projective $R$ module. Since $L$ is isomorphic to $K \otimes S$, the rank of $S$ as a projective $R$ module is $d$, the dimension of $L$. If we knew that $S$ splits $I$, then by Theorem 3 we would know $(I)^{d}=1$ in Pic $(R)$ and thus $d \geqq n$, the order of $(I)$. Hence we would have $d=n$, which is what we wanted.

Since $S$ is a projective $R$-module, $S \otimes I$ is $S$-isomorphic to $J=S I$. Let $v$ be any root of $g(X)$ in $L$. Then $J^{n}=S w=(S v)^{n}$ and thus since $S$ is Dedekind [10, p. 281], $J=S v$ and so $S$ splits $I$.

Now suppose $n$ is a unit of $R$. By Propositions 6 and 4, we know $T$ is isomorphic to a separable $R$-order $T^{\prime}$ of $L$. Since $T^{\prime}$ is separable, it is maximal, i.e., $T^{\prime}=S$, the integral closure of $R$ in $L$ and thus is Dedekind.

Now let us do the case in which $n$ is the zero element of $R$. All we have to show is that $T$ is a domain since clearly $n$ is not a unit. By Proposition 6 we need to show that $X^{n}-w$ is irreducible over $K$. Suppose $p$ is the characteristic of $K$ and $n_{1}=p^{e}$ is the highest power of $p$ dividing $n$. Let $n_{2}=n / n_{1}$. By Lemma 4 , we 
need to show that both $X^{n_{2}}-w$ and $X^{n_{2}}-w$ are irreducible. Let $I^{\prime}=I^{n_{1}}$. Then clearly $I^{\prime n_{2}}=R w, n_{2}$ is the order of $\left(I^{\prime}\right)$ in Pic $(R)$ and $n_{2}$ is nonzero in $R$. Hence by the proof of the theorem in the special case, $X^{n_{2}}-w$ is irreducible. Now we note that $w$ cannot be a $p$-th power in $K$. Otherwise if $w=v^{p}$ for some $v$ in $K$, and if $n^{\prime}=n / p$, then $I^{n^{\prime} p}=(R v)^{p}$ indicates $I^{n^{\prime}}=R v$ and so $n$ would not be the order of $(I)$. Since $w$ is not a $p$-th power, $X-w$ is irreducible $[10$, p. 65].

Suppose $I$ is a nonzero ideal of the Dedekind domain $R$ with $I^{n}=R w$, and let $T=T_{n}\left(I^{-1} / R, w\right)$ and let $S$ be the integral closure of $R$ in $L=K[X] /\left(X^{n}-w\right)$. Then Theorem 4 gives sufficient conditions to ensure that $T$ is a domain or is equal to $S$. We conclude with two examples illustrating then if the hypotheses of Theorem 4 do not hold, the conclusion may or may not still hold.

EXAMPLE $1 . \quad n$ is neither a unit of $R$ nor the order of $I$, but $T=S$ and is a Dedekind domain.

Proof. Let $R=I=Z$-the rational integers. Let $n=2$ and $w=-1$. Clearly $T=Z[i]$-the Gaussian integers in $L=Q[i]$.

Example 2. $n$ is the order of $(I)$ in Pic $(R)$, is a non zero divisor but is not a unit of $R$ and $T \neq S$.

Proof. Let $p$ and $q$ be distinct rational primes with $p \equiv q \equiv 1$ $(\bmod 4)$. Let $R=Z[\sqrt{-p q}]$ and $I=R p+R \sqrt{-p q}$. One can check that $R$ is the integral closure of $Z$ in $Q(\sqrt{-p q})$ and is thus Dedekind and that $I$ is nonprincipal with $I^{2}=R p$. Let $n=2$ and let $w=$ $\pm p$. It is not too difficult to show that $L$ is an unramified field extension of $K$ and thus $T$ is a domain and $S$ is separable [5, p. 21]. Since 2 is not a unit of $R$, by Proposition 4 we know that $T$ is not separable; hence $T \neq S$.

\section{REFERENCES}

1. S. A. Amitsur, Generic splitting fields of central simple algebras, Ann. of Math. 62 (1955), 8-43.

2. H. Bass, Lectures in Algebraic K-theory, Tata Institute of Fundamental Research, Bombay, 1967.

3. N. Bourbaki, Algebre Commutative, Chapter 1, Hermann, Paris, 1961.

4. H. Cartan and S. Eilenberg, Homological Algebra, Princeton Univ. Press, Princeton, 1956.

5. S. Chase and A. Rosenberg, Galois theory and Galois cohomology of commutative rings, Amer. Math. Soc. Memoirs, No. 52, 1965.

6. M. Deuring, Algebren (Zweite Auflage), Springer-Verlag, New York, 1968. 
7. T. Nagahara, A note on Galois theory of commutative rings, Proc. Amer. Math. Soc. 18 (1967), 334-340.

8. P. Roquette, On the Galois cohomology of the projective linear group and its applications to the construction of generic splitting fields of algebras, Math. Ann. 150 (1963), 411-439.

9. A. Rosenberg and D. Zelinsky, Automorphisms of separable algebras, Pacific J. Math. 11 (1961), 1109-17.

10. O. Zariski and P. Samuel, Commutative Algebra, Volume 1, D. Von Nostrand, Princeton, 1958.

Received March 10, 1970.

UNIVERSITY OF ILLINOIS

URBANA, ILLINOIS 


\section{PACIFIC JOURNAL OF MATHEMATICS}

\section{EDITORS}

\author{
H. SAMELSON \\ Stanford University \\ Stanford, California 94305

\section{Richard Pierce} \\ University of Washington \\ Seattle, Washington 98105
}

J. DugundJI

Department of Mathematics

University of Southern California

Los Angeles, California 90007

RichaRd ARENS

University of California

Los Angeles, California 90024

\section{ASSOCIATE EDITORS}
E. F. BECKENBACH
B. H. NeUMANN
F. WOLE
K. YoshidA

\section{SUPPORTING INSTITUTIONS}

\author{
UNIVERSITY OF BRITISH COLUMBIA \\ CALIFORNIA INSTITUTE OF TECHNOLOGY \\ UNIVERSITY OF CALIFORNIA \\ MONTANA STATE UNIVERSITY \\ UNIVERSITY OF NEVADA \\ NEW MEXICO STATE UNIVERSITY \\ OREGON STATE UNIVERSITY \\ UNIVERSITY OF OREGON \\ OSAKA UNIVERSITY \\ UNIVERSITY OF SOUTHERN CALIFORNIA
}

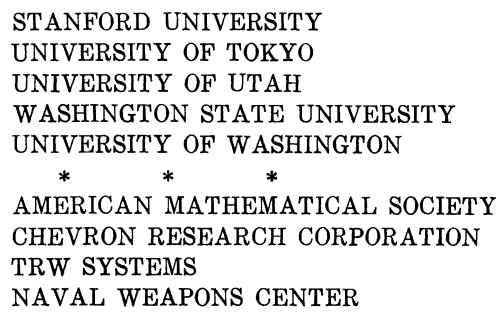

The Supporting Institutions listed above contribute to the cost of publication of this Journal, but they are not owners or publishers and have no responsibility for its content or policies.

Mathematical papers intended for publication in the Pacific Journal of Mathematics should be in typed form or offset-reproduced, (not dittoed), double spaced with large margins. Underline Greek letters in red, German in green, and script in blue. The first paragraph or two must be capable of being used separately as a synopsis of the entire paper. The editorial "we" must not be used in the synopsis, and items of the bibliography should not be cited there unless absolutely necessary, in which case they must be identified by author and Journal, rather than by item number. Manuscripts, in duplicate if possible, may be sent to any one of the four editors. Please classify according to the scheme of Math. Rev. Index to Vol. 39. All other communications to the editors should be addressed to the managing editor, Richard Arens, University of California, Los Angeles, California, 90024.

50 reprints are provided free for each article; additional copies may be obtained at cost in multiples of 50 .

The Pacific Journal of Mathematics is published monthly. Effective with Volume 16 the price per volume (3 numbers) is $\$ 8.00$; single issues, $\$ 3.00$. Special price for current issues to individual faculty members of supporting institutions and to individual members of the American Mathematical Society: $\$ 4.00$ per volume; single issues $\$ 1.50$. Back numbers are available.

Subscriptions, orders for back numbers, and changes of address should be sent to Pacific Journal of Mathematics, 103 Highland Boulevard, Berkeley, California, 94708.

PUBLISHED BY PACIFIC JOURNAL OF MATHEMATICS, A NON-PROFIT CORPORATION

Printed at Kokusai Bunken Insatsusha (International Academic Printing Co., Ltd.), 7-17, Fuj̣imi 2-chome, Chiyoda-ku, Tokyo, Japan. 


\section{Pacific Journal of Mathematics}

\section{Vol. 35, No. $2 \quad$ October, 1970}

Valentin Danilovich Belousov and Palaniappan L. Kannappan, Generalized Bol functional equation .................................... 259

Charles Morgan Biles, Gelfand and Wallman-type compactifications ........... 267

Louis Harvey Blake, A generalization of martingales and two consequent convergence theorems .................................... 279

Dennis K. Burke, On p-spaces and $w \Delta$-spaces..................... 285

John Ben Butler, Jr., Almost smooth perturbations of self-adjoint operators . . . . . . 297

Michael James Cambern, Isomorphisms of $C_{0}(Y)$ onto $C(X) \ldots \ldots \ldots \ldots \ldots . \ldots 307$

David Edwin Cook, A conditionally compact point set with noncompact closure ... 313

Timothy Edwin Cramer, Countable Boolean algebras as subalgebras and homomorphs .........................................

John R. Edwards and Stanley G. Wayment, A v-integral representation for linear operators on spaces of continuous functions with values in topological vector spaces.............................................

Mary Rodriguez Embry, Similarities involving normal operators on Hilbert

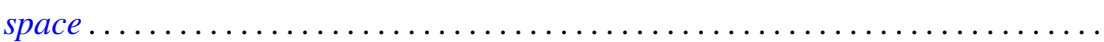

Lynn Harry Erbe, Oscillation theorems for second order linear differential

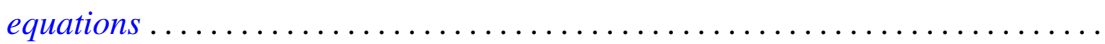

William James Firey, Local behaviour of area functions of convex bodies .......... Joe Wayne Fisher, The primary decomposition theory for modules ..............

Gerald Seymour Garfinkel, Generic splitting algebras for Pic ..................

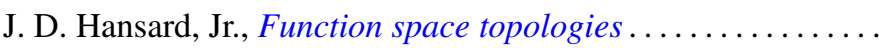

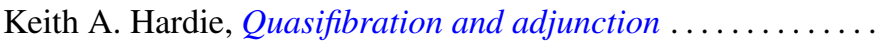

G. Hochschild, Coverings of pro-affine algebraic groups ...........

Gerald L. Itzkowitz, On nets of contractive maps in uniform spaces ..

381

389

399

417

Melven Robert Krom and Myren Laurance Krom, Groups with free nonabelian subgroups....................................

James Robert Kuttler, Upper and lower bounds for eigenvalues by finite differences ......................................

Dany Leviatan, A new approach to representation theory for convolution transforms . . .

Richard Beech Mansfield, Perfect subsets of definable sets of real numbers ...

Brenda MacGibbon, A necessary and sufficient condition for the embedding of a

Lindelof space in a Hausdorff $\mathscr{H} \sigma$ space ..................

David G. Mead and B. D. McLemore, Ritt's question on the Wronskian ....

Edward Yoshio Mikami, Focal points in a control problem .....

Paul G. Miller, Characterizing the distributions of three independent n-dimensional random variables, $X_{1}, X_{2}, X_{3}$, having analytic characteristic functions by the joint distribution of $\left(X_{1}+X_{3}, X_{2}+X_{3}\right)$. . .

P. Rosenthal, On the Bergman integral operator for an elliptic partial differential equation with a singular coefficient....

Douglas B. Smith, On the number of finitely generated $O$-group 